\title{
Cosmic-ray Propagation in Light of Recent HAWC Observations of Pulsar Wind Nebula
}

\section{Guðlaugur Jóhannesson}

Science Institute, University of Iceland \& Nordita, KTH Royal Institute of Technology and Stockholm University

E-mail: gudlauguehi.is

\section{Troy A. Porter*}

W. W. Hansen Experimental Physics Laboratory and Kavli Institute for Particle Astrophysics and Cosmology, Stanford University

E-mail: tporterestanford.edu

\section{Igor V. Moskalenko}

W. W. Hansen Experimental Physics Laboratory and Kavli Institute for Particle Astrophysics and Cosmology, Stanford University

E-mail: imosestanford.edu

\begin{abstract}
Recent observations made with the High Altitude Water Cherenkov (HAWC) telescope of pulsar wind nebula (PWN) suggest that the diffusion in their vicinity is characterised with a lower diffusion constant than that predicted for propagation in the interstellar medium from observations of cosmic-ray (CR) fluxes at Earth. In this contribution, it is shown that models with the slow diffusion region localised about PWN can successfully explain the HAWC observations of the Geminga PWN and still retain consistency with other CR measurements. Parameter exploration shows that the size of the smaller diffusion zone has implications for the both the PWN emission at lower energies observable by the Fermi Large Area Telescope and the predicted positron flux at the Earth. Unless the Geminga PWN is unique, there are likely many small regions with slow diffusion throughout the Milky Way. The consequences for the propagation of CRs and the resulting interstellar emissions from across the Galaxy are discussed and explored.
\end{abstract}

36th International Cosmic Ray Conference -ICRC2019-

July 24th - August 1st, 2019

Madison, WI, U.S.A.

${ }^{*}$ Speaker. 


\section{Introduction}

Cosmic rays (CRs) propagating through the interstellar medium scatter off magnetic turbulences in a process that on large scales can be described with a diffusive transport equation with a rigidity dependent diffusion coefficient (Ginzburg \& Syrovatskii, 1964; Berezinskii et al., 1990). Observations of the ratios of stable secondary to primary species in CRs and the abundances of radioactive secondaries are usually employed to determine both the power-law index and normalization of the diffusion coefficient averaged over a significant volume of the interstellar space surrounding the Solar system (e.g., Strong \& Moskalenko, 1998; Strong et al., 2007; Jóhannesson et al., 2016), typically several kpc in radius. Recent estimates of the power-law index and normalization vary somewhat depending on the details of the propagation models. Values of the index between $\sim 0.2$ and $\sim 0.6$ are found with a normalization of a few times $10^{28} \mathrm{~cm}^{2} \mathrm{~s}^{-1}$ at a rigidity of 4 GV (e.g., Yuan et al., 2017; Génolini et al., 2017; Boschini et al., 2018).

Recent observations of the extended TeV emission around Geminga and PSR B0656+14 PWN by the High Altitude Water Cherenkov (HAWC) telescope constrain the diffusion coefficient in their vicinities to be about two orders of magnitude smaller than the average value derived from observations of CRs (Abeysekara et al., 2017). Application of such slow diffusion to the local Milky Way results in a contradiction with other CR observations, in particular observations of highenergy CR electrons and positrons. Fast energy losses of $\mathrm{TeV}$ particles through inverse Compton (IC) scattering and synchrotron emission limits their lifetime to $\sim 100$ ky (Strong \& Moskalenko, 1998). If such slow diffusion is representative for the ISM within about a few hundred pc of the Solar system, the sources of the $\mathrm{TeV}$ particles detected at Earth also need to be within a few $10 \mathrm{~s}$ of pc. Profumo et al. (2018) highlighted that such nearby sources have not been identified and proposed a two-zone diffusion model with the slow diffusion confined to a small region around the PWN. Other authors have considered similar scenarios with varying details (Tang \& Piran, 2018; Fang et al., 2018; Evoli et al., 2018).

In this contribution the two-zone diffusion model is explored using the latest version of the GALPROP $^{1}$ propagation code (Porter et al., 2017; Jóhannesson et al., 2018). The results indicate that such a model is a viable interpretation for the HAWC observations and confirm similar conclusions made by other authors. Predictions for lower-energy $\gamma$-ray emission that can be observed with the Fermi Large Area Telescope (Fermi-LAT) are made and the contribution of energetic positrons coming from Geminga to the observed CR positron flux in different scenarios is studied. Unless both Geminga and PSR B0656+14 are special cases there are expectations for more regions of slower diffusion around other PWNe in the Milky Way. The implications that such inhomogeneity of the diffusion in the ISM can have on the CR distribution throughout the Milky Way is also explored. This work has also been published in Jóhannesson et al. (2019) which provides more details.

\section{Two zone model for Geminga}

It is assumed that the Geminga pulsar is injecting accelerated electrons and positrons into the ISM in equal numbers with a fraction $\eta$ of its spin-down power converted to pairs. After

\footnotetext{
${ }^{1} \mathrm{http}: / /$ galprop.stanford.edu
} 
injection the particles propagate via a diffusive process. The energy distribution of the injected electrons/positrons is described with a smoothly joined broken power-law with low energy index $\gamma_{0}$ and high energy indes $\gamma_{1}$. The smoothness parameter is assumed constant and so is the low-energy index $\gamma_{0}=-1$ and the break energy $E_{b}=10 \mathrm{GeV}$. This low-energy break effectively truncates the injected particle spectrum at energies below that explored in this paper and has no effect on the results. The formulation for the spin-down power is identical to that of Abeysekara et al. (2017). The distance to Geminga has been determined to be $250 \mathrm{pc}$ and its proper motion to be 107.5 mas

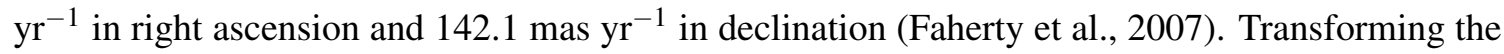

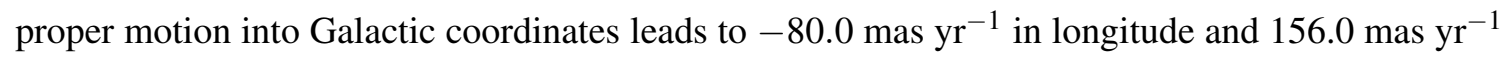
in latitude. In this case, Geminga was born in a SN explosion of a star that has travelled from the direction of the Orion OB1a association (Smith et al., 1994). While the $\mathrm{TeV} \gamma$-ray emission is not significantly affected by the proper motion, the large traveled distance and proximity of Geminga to the Sun should produce a "trailing" tail of CRs whose $\gamma$-ray emissions may be observable at lower energies with Fermi-LAT.

For the two-zone diffusion model, the diffusion coefficient in a confined region around the pulsar within a radius of $r_{z}$ is assumed to be lower than that in the ISM. This region will hereafter be called a "slower diffusion zone" (SDZ). It is also assumed that the rigidity dependence of the diffusion coefficient is the same for the SDZ and the ISM. The diffusion coefficient is normalized at a rigidity of $4 \mathrm{GV}$, having a value of $D_{z}$ within $r_{z}$, rising exponentially to a value of $D_{0}$ at a radius of $r_{t}$ in a transition zone and then staying constant at a value of $D_{0}$ outside $r_{t}$. Depending on the exact origin of the SDZ, its radius can be time dependent as can its location. To be consistent with the HAWC observations, both $r_{z}$ and $r_{t}$ have to be of the order of a few tens of pc at the current time.

Two general categories distinguished by the origin of the SDZ are considered. For the first category the SDZ is due to events external to the PWN itself (external SDZ). It is assumed that the particle propagation time in the zone is much longer than the time necessary to generate such a zone itself ("instant" generation) and its location is fixed. For the second category, the SDZ is assumed to be associated with and generated by the PWN itself (PWN SDZ). Therefore, the SDZ is moving with the Geminga pulsar and its size increases proportionally to the square root of time in a diffusive manner. In this case, the possibility that there is a momentum transfer from the pulsar to the ISM is considered. Using conservation of momentum and mass it can be shown that the velocity of the pulsar evolves as

$$
v=\frac{v_{0}}{\left(3 A_{d} v_{0} t^{2} / 2+1\right)^{2 / 3}},
$$

where $A_{d}$ is a drag coefficient that is linearly related to the density of the ISM that is swept up by the SDZ. $A_{d}$ also depends on the rate of change of the size of the SDZ. Using realistic assumptions for both results in an initial velocity of Geminga of few hundred $\mathrm{km} / \mathrm{s}$ and total distance traveled of $\sim 100 \mathrm{pc}$.

The calculations are made using the latest release of the GALPROP code $^{1}$ (Porter et al., 2017; Jóhannesson et al., 2018). The values of $D_{0}=4.5 \times 10^{28} \mathrm{~cm}^{2} \mathrm{~s}^{-1}$ and $\delta=0.35$ are chosen to match the latest AMS-02 data. The SDZ diffusion coefficient of $D_{z}=1.3 \times 10^{26} \mathrm{~cm}^{2} \mathrm{~s}^{-1}$ at $R_{0}=4 \mathrm{GV}$ corresponds to the value derived from the HAWC observations at higher energies Abeysekara et al. 

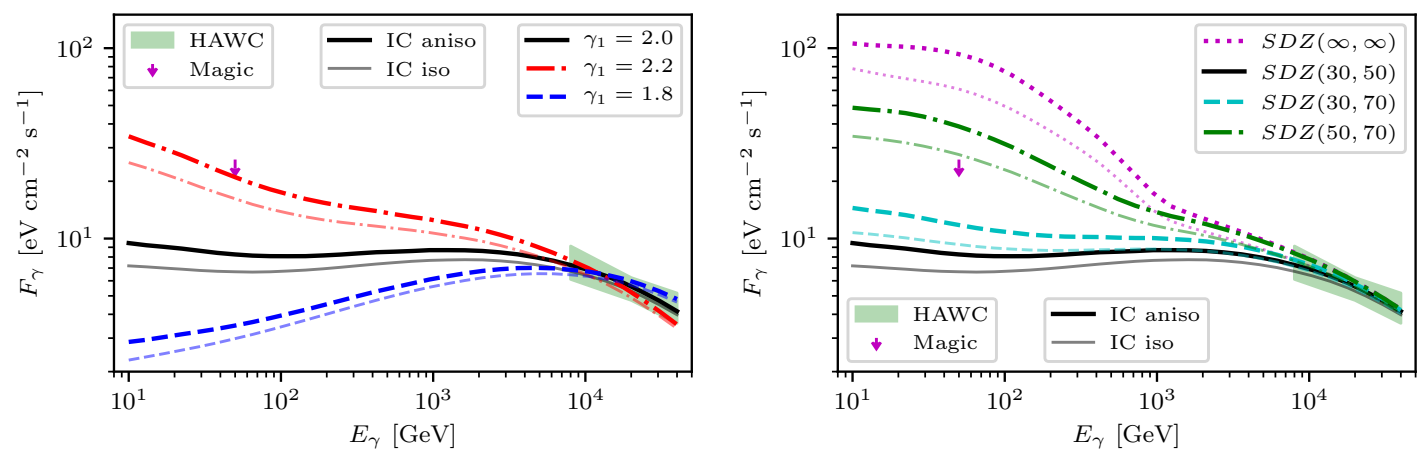

Figure 1: Spectrum of IC emission averaged over a $10^{\circ}$ wide region around the current location of Geminga. The models shown here assume Geminga is stationary and that the SDZ is stationary and centered at Geminga. The left panel shows the spectrum for different values of $\gamma_{1}$ as indicated in the legend, but using fixed values of $\left(r_{z}, r_{t}\right)=(30,50) \mathrm{pc}$. The right panel shows the spectrum for models with different pairs of $\left(r_{z}, r_{t}\right)$, but fixed value of $\gamma_{1}=2.0$. The green shaded region corresponds to HAWC observations assuming a diffusion profile for the spatial distribution (Abeysekara et al., 2017) and the magenta arrow is the upper limit from observations with the MAGIC telescope corrected for the diffusion profile (Tang \& Piran, 2018). The thin lines are for models using the isotropic IC assumption.

(2017). The calculations also include diffusive re-acceleration with an Alfvén speed $v_{A}=17 \mathrm{~km}$ $\mathrm{s}^{-1}$, as determined from the fit to the secondary-to-primary data.

For a comparison with results of previous studies the first set of calculations is performed assuming that there is no proper motion of Geminga. The SDZ is centered at the current location of Geminga $\left(l_{G}, b_{G}\right)=(195.14,4.26)$ and is static in size. Two sets of calculations are performed, the first having $\gamma_{1}=2.0$ fixed but using several combinations of $\left(r_{z}, r_{t}\right)$, covering a range from $30 \mathrm{pc}$ to $50 \mathrm{pc}$ for $r_{z}$ and from $50 \mathrm{pc}$ to $70 \mathrm{pc}$ for $r_{t}$. Calculations are also made using a model with $\left(r_{z}, r_{t}\right)=(\infty, \infty)$ for a comparison with the results from Abeysekara et al. (2017). The second set has $\left(r_{z}, r_{t}\right)=(50,50)$ pc fixed, but uses a few values for $\gamma_{1}$. The efficiency $\eta$ is adjusted in each case to match the surface brightness profile observed by HAWC.

The average spectrum of the IC emission over a circular region of $10^{\circ}$ in radius around the current location of Geminga is shown in Figure 1 for both sets of calculations. The models are all tuned to agree with the HAWC data and, therefore, the predicted profiles differ significantly at lower energies. Shown is the prediction made using both the full IC cross section as well as the isotropic assumption for the IC cross section (thin curves). The isotropic IC approximation has a significant effect on the model prediction. The perceived degeneracy between the size of the SDZ and the injected spectrum of the particles from this figure is broken when the angular profile of the low-energy emission is taken into account. Changing the injection spectrum will not affect the shape of the angular profile while changing the SDZ size does. The angular extent of the emission should therefore be a good indicator of the size of the SDZ.

One of the main conclusions of the study by Abeysekara et al. (2017) is that the unexpected rise in the positron fraction as measured recently by AMS-02 (Aguilar et al., 2014) cannot be due to the positrons accelerated by the Geminga PWN. This conclusion is based on a one-zone diffusion model constructed to agree with the HAWC data. Figure 2 shows the positron flux at Earth as 

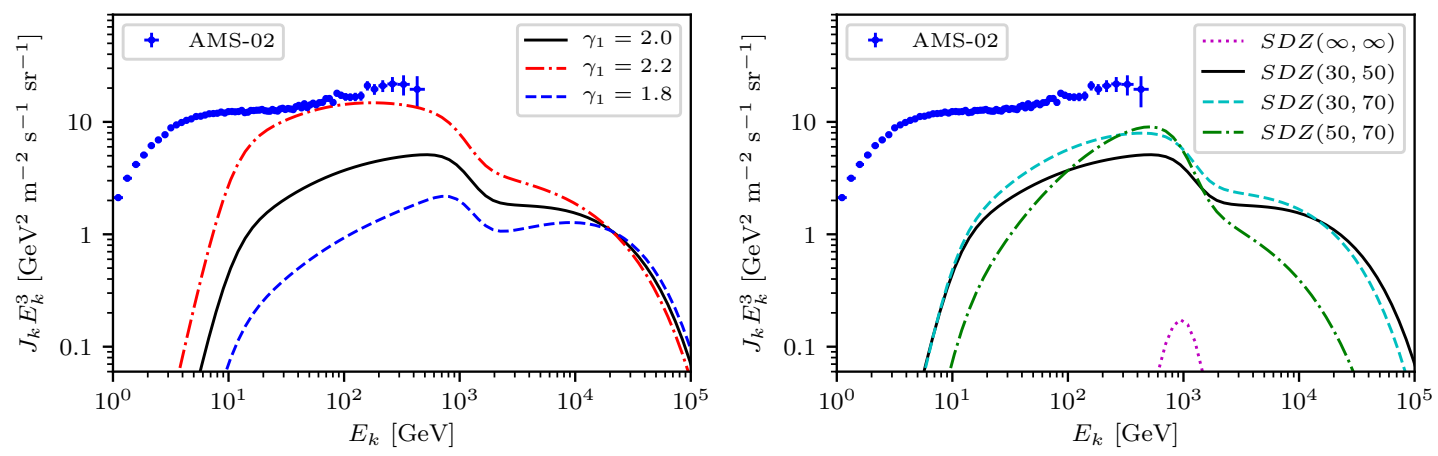

Figure 2: Predicted flux of positrons at Earth. The models shown here assume Geminga is stationary and that the SDZ is stationary and centered at the current location of Geminga. The left panel shows the spectrum for different values of $\gamma_{1}$ as indicated in the legend, but using a fixed SDZ size $\left(r_{z}, r_{t}\right)=(30,50) \mathrm{pc}$. The right panel shows the spectrum for models with different pairs of $\left(r_{z}, r_{t}\right)$, but fixed value of $\gamma_{1}=2.0$. The points are AMS-02 data (Aguilar et al., 2014).
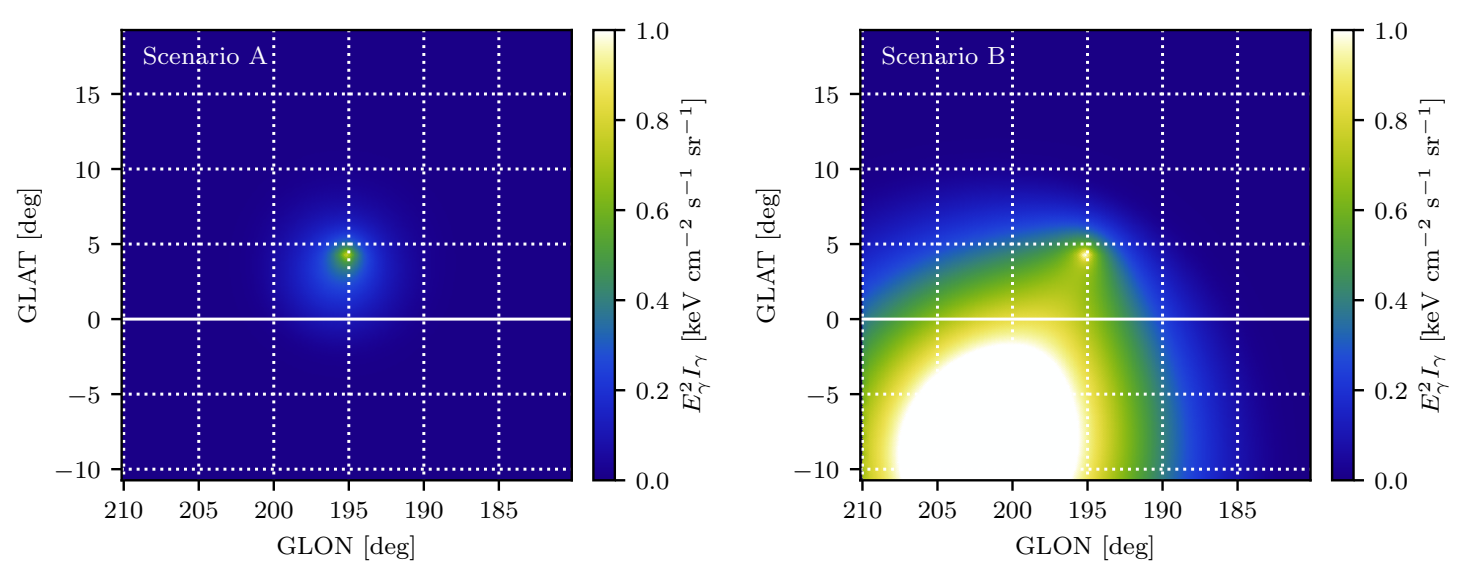

Figure 3: IC intensity maps evaluated at $10 \mathrm{GeV}$ around the current location of Geminga. Shown are maps for scenarios A (left) and B (right). The maps for scenarios C and D are very similar to that of A.

predicted by the models considered in this paper, where the different panels illustrate models with different SDZ sizes, and the effect of changing $\gamma_{1}$, respectively. The results clearly show a strong variation in the expected positron flux at Earth. A smaller SDZ leads to larger flux of positrons at Earth because of the larger effective diffusion coefficient. Only if the SDZ extends all the way to the solar system are the results of Abeysekara et al. (2017) reproduced. Larger values of $\gamma_{1}$ also results in a larger positron flux at Earth in the observed energy range, meanwhile values larger than $\gamma_{1} \approx 2.2$ are excluded as the predicted positron flux would exceed the data for a SDZ of size $\left(r_{z}, r_{t}\right)=(30,50) \mathrm{pc}$.

So far the calculations have been made considering a stationary source located at the current position of Geminga $\left(l_{G}, b_{G}\right)$. However, such an approximation is not supported by its observed large proper motion. In the following analysis, the proper motion is taken into account, but different assumptions are made on the origin of the SDZ and the value of the drag coefficient $A_{d}$ introduced in Eq. (2.1). The different assumptions are referred to as scenarios A to D and detailed below. 

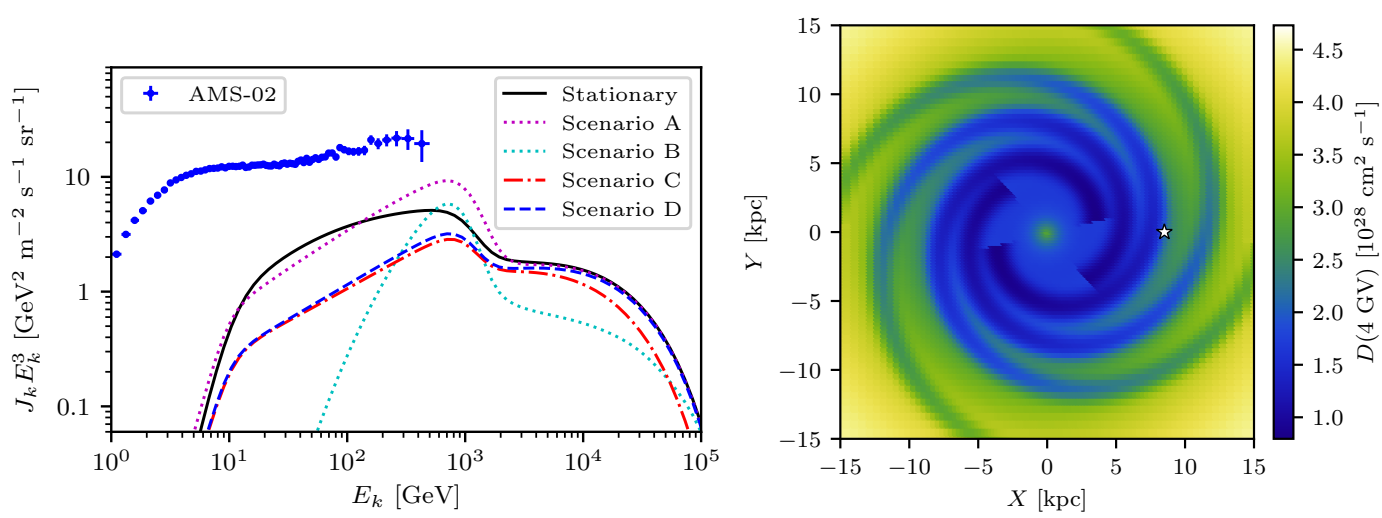

Figure 4: Left panel: predicted positron fluxes at Earth for scenarios A through D. Also shown are the results for a model where Geminga is stationary with $\left(r_{z}, r_{t}\right)=(30,50) \mathrm{pc}$ and $\gamma_{1}=2.0$. The points are AMS-02 data (Aguilar et al., 2014). Right panel: diffusion coefficient evaluated in the Galactic plane at the normalization rigidity $R_{0}=4 \mathrm{GV}$ for a model with the SA50 source distribution where the effective diffusion coefficient is given by Eq. (3.1). The location of the Sun is marked as a white star and the GC is at $(0,0)$.

For scenarios $\mathrm{A}$ and $\mathrm{B}$, the $\mathrm{SDZ}$ is assumed to be stationary and is unrelated to Geminga. In both scenarios, $A_{d}=0$ and the pulsar velocity is constant. Scenario A assumes that the SDZ is centered on the current position of Geminga by chance, and that the parameters of the zone are: $\left(r_{z}, r_{t}\right)=(30,50)$ pc. Scenario B assumes that the SDZ is centered at the birth place of Geminga. In this scenario the SDZ has to be much larger to extend from the birth place of Geminga to its current location, $\left(r_{z}, r_{t}\right)=(90,110) \mathrm{pc}$. For scenarios $\mathrm{C}$ and $\mathrm{D}$, the center of the SDZ follows the location of Geminga and its size increases proportionally to the square root of time, normalized such that the final size of the SDZ is $\left(r_{z}, r_{t}\right)=(30,50) \mathrm{pc}$. The difference between these two scenarios is the value of the drag coefficient, $A_{d}$. In scenario $\mathrm{C}, A_{d}=0$ and the velocity is constant over time, while in scenario $\mathrm{D}, A_{d}=2.636 \times 10^{-8} \mathrm{pc}^{-1} \mathrm{yr}^{-1}$ giving Geminga an initial velocity of $410 \mathrm{~km} \mathrm{~s}^{-1}$. For scenarios A through D, $\gamma_{1}=2.0$ is fixed. The effect of varying the size of the SDZ and value of $\gamma_{1}$ is similar to that of the stationary Geminga.

The surface profile predicted by the models at $\mathrm{TeV}$ energies is fully compatible with the HAWC observation and not strongly affected by the proper motion of Geminga. In contrast, at lower energies all models produce quite different distributions of the surface brightness. In particular, considerable asymmetry in the intensity maps around $10 \mathrm{GeV}$ is seen in Figure 3, especially for scenario B with the largest SDZ. All scenarios where Geminga is moving show a distinctive tail in the low energy range. In the extreme case of scenario B the tail is exceptionally broad and the emission of the tail is, in fact, much brighter than that at the current location of Geminga. The differences between other scenarios are small and hard to distinguish. Observations of the Geminga PWN at $10 \mathrm{GeV}$ will thus hardly be able to distinguish between these scenarios, but may be able to verify the presence of the tail in Scenario B.

Despite their similar $\gamma$-ray emission maps (with exception of scenario B), all scenarios predict different positron fluxes at Earth (Figure 4, left panel). For energies below $1 \mathrm{TeV}$, the positron flux in scenario $\mathrm{A}$ is about a factor of 5 larger than that predicted for scenarios $\mathrm{C}$ and $\mathrm{D}$. The difference between scenarios $\mathrm{C}$ and $\mathrm{D}$ is much smaller and mostly at the highest energies where scenario $\mathrm{D}$ 
produces a higher flux. This is due to the faster movement of the diffusion zone in scenario D that allows the CR particles to escape quickly once the diffusion zone has left them behind. This is somewhat idealized and it is more likely that the SDZ will have a shape elongated along the direction of motion. For such a case the results would become closer to scenario B, with a longer IC emission tail at low energies and smaller flux of positrons at low and high energies, but with a larger peak at around $300 \mathrm{GeV}$.

\section{Implications for CR propagation in the Milky Way}

If the result for the two PWNe reported in Abeysekara et al. (2017) are not special cases, then it is likely that there are similar pockets of slow diffusion around many CR sources elsewhere in the Milky Way. The effective diffusion coefficient would thus be smaller in regions where the number density of CR sources is higher. Here it is assumed that the distribution of SDZs follows that of the CR sources, while the exact origin of these SDZs is not essential. With current CR propagation codes and reasonable resources resolving the entire Milky Way with a few pc grid size on short time scales is intractable. An alternative approach is therefore needed. The effect of an SDZ is a local increase of the density of CRs for a certain period of time and the CRs thus spend more time in the vicinity of their sources compared to a model with a homogeneous diffusion. This is equivalent to an effective decrease of the diffusion coefficient averaged over a larger volume. Even though, such an approximation does not account for the detailed spatial distribution of the individual SDZs, it does account for the increased interaction rate with the ISM, such as cooling and generation of secondary CR particles. Therefore, this approximation should still provide for the correct spectra and abundances of CR species.

Assuming each CR source contains a SDZ, it is possible to show (Jóhannesson et al., 2019) that

$$
D(\vec{x})=D_{0}\left\{1+\left[q(\vec{x}) V_{z}\right]^{2 / 3} \frac{D_{0}}{D_{z}}\right\}^{-1}
$$

is the appropriate equation for the effective diffusion coefficient. Here $q(\vec{x})$ is the CR source number density and $V_{z}$ the volume of the diffusion zone. Assuming further that there are about 1000 active CR sources in the Milky Way and that each has a radius of $30 \mathrm{pc}$, the total volume of the SDZ is about $0.1 \mathrm{kpc}^{3}$. To test the effect of the modified diffusion, the propagation parameters of two different CR source distributions were tuned to match CR data observed with the AMS-02 instrument. The distributions used were SA0 and SA50 from Porter et al. (2017). SA0 is a 2D disk model while SA50 has half of the total number of CR sources distributed in spiral arms while the other half is a disk. The propagation parameters of the models were tuned with a homogeneous diffusion and the modified effective diffusion. The distribution of the effective diffusion coefficient $D(\vec{x})$ in the Galactic plane for the SA50 model is shown in the right panel of Figure 4, where the numbers correspond to its value at the normalization rigidity $R_{0}=4 \mathrm{GV}$.

The best fit propagation parameters are similar for all models and the modified diffusion and different source distributions only slightly affect the best-fit values. Most interestingly, despite the fact that the value of the effective diffusion coefficient exhibits strong spatial variations in the Galactic plane including at the Solar system location, where it is about a factor of 2 smaller compared to that in the model with homogeneous diffusion (Fig. 4), the value of $D_{0}$ is not significantly 
different from that obtained for the homogeneous model. This is connected with the relatively small volume affected by the modified diffusion regions because they are associated with the CR sources, which have a relatively narrow distribution about the Galactic plane with exponential $z$ scale-height $200 \mathrm{pc}$. The modified diffusion slightly affects the low-energy part of the spectrum, resulting in smaller modulation potentials and Alfvén speeds. Overall the addition of the SDZs does not significantly alter the global diffusive properties of the ISM.

\section{Acknowledgments}

GALPROP development is partially funded via NASA grant NNX17AB48G.

\section{References}

Abeysekara, A. U., Albert, A., Alfaro, R., et al. 2017, Science, 358, 911

Aguilar, M., Aisa, D., Alvino, A., et al. 2014, Physical Review Letters, 113, 121102

Berezinskii, V. S., Bulanov, S. V., Dogiel, V. A., \& Ptuskin, V. S. 1990, Astrophysics of cosmic rays

Boschini, M. J., Della Torre, S., Gervasi, M., et al. 2018, ApJ, 858, 61

Evoli, C., Linden, T., \& Morlino, G. 2018, Phys. Rev. D, 98, 063017

Faherty, J., Walter, F. M., \& Anderson, J. 2007, Ap\&SS, 308, 225

Fang, K., Bi, X.-J., Yin, P.-F., \& Yuan, Q. 2018, ApJ, 863, 30

Génolini, Y., Serpico, P. D., Boudaud, M., et al. 2017, Physical Review Letters, 119, 241101

Ginzburg, V. L., \& Syrovatskii, S. I. 1964, The Origin of Cosmic Rays

Jóhannesson, G., Porter, T. A., \& Moskalenko, I. V. 2018, ApJ, 856, 45

Jóhannesson, G., Porter, T. A., \& Moskalenko, I. V. 2019, ApJ, 879, 91

Jóhannesson, G., Ruiz de Austri, R., Vincent, A. C., et al. 2016, ApJ, 824, 16

Porter, T. A., Jóhannesson, G., \& Moskalenko, I. V. 2017, ApJ, 846, 67

Profumo, S., Reynoso-Cordova, J., Kaaz, N., \& Silverman, M. 2018, Phys. Rev. D, 97, 123008

Smith, V. V., Cunha, K., \& Plez, B. 1994, A\&A, 281, L41

Strong, A. W., \& Moskalenko, I. V. 1998, ApJ, 509, 212

Strong, A. W., Moskalenko, I. V., \& Ptuskin, V. S. 2007, Annual Review of Nuclear and Particle Science, 57, 285

Tang, X., \& Piran, T. 2018, ArXiv e-prints, arXiv:1808.02445

Yuan, Q., Lin, S.-J., Fang, K., \& Bi, X.-J. 2017, Phys. Rev. D, 95, 083007 